\title{
Physicochemical characterization and formation of glyphosate-laden nano-emulsion for herbicide formulation.
}

\begin{abstract}
A nano-emulsion system was developed for pesticide formulation. Pseudoternary phase diagrams were constructed consisting emulsion system of long-chain fatty acid methyl esters (LFAMEs)/mixed surfactant/water and a quarternary component, glyphosate isopropylamine (IPA) as a herbicide active. Isotropic (L) regions were formed in the phase diagrams using mixed surfactant long-chain alkylpolyglucosides (LAPG) and ethoxylated -(3hydroxypropyl)-heptamethyltrisiloxane (organosilicone) at the ratios of 9:1, 8:2 and 7:3. Preformulation concentrates were chosen from the L regions with less than $20 \%$ (w/w) of inerts (LFAMEs + mixed surfactant) and were characterized with regard to particle size, particle aging rate and thermostability study. A pre-formulation concentrate with the lowest aging rate and stable at high temperature $\left(54{ }^{\circ} \mathrm{C}\right)$ was selected for the mechanisms study of the preformulation concentrate in conjunction with the development of nano-emulsion formulation. The transmission electron microscopy (TEM) result showed that the pre-formulation concentrate appeared as a polymerized multi-connected network. Upon water dilution of the pre-formulation concentrate with gentle stirring (low-energy emulsification method), welldispersed nanoparticles were formed with no needle structure being observed. The nanoemulsion particles were incorporated well with the glyphosate IPA thus inferring that this nano-emulsion system could ameliorate the bioactivity and bioavailability of the herbicide.
\end{abstract}

Keyword: Nano-emulsion; Glyphosate isopropylamine; Alkylpolyglucosides; Pesticide formulations; Low-energy emulsification 